\title{
La comunicación directa entre los diputados del Congreso y los ciudadanos a través del correo electrónico. Nuevos hábitos de información y participación ciudadana en la VIII Legislatura de las Cortes Generales (2004-2008)
}

\section{Direct communication between Members of Congress and citizens via e-mail. New habits of information and citizen participation in the Eighth Legislature of the Spanish Parliament (2004-2008)}

\author{
Eva Campos Domínguez. Universidad de Valladolid
}

Recibido: 28-01-2010 - Aceptado: 30-IV-2010

Resumen:

Las nuevas tecnologías de la información, entre las que figura como máximo estandarte Internet, han extendido la idea de que políticos y ciudadanos podrían comunicarse directamente utilizando algunas de las herramientas más populares, como lo es el correo electrónico. Este artículo recoge los principales resultados de una investigación que tiene como objetivo averiguar el índice de respuesta que puede tener un ciudadano común al enviar un correo electrónico a los diputados del Congreso en una perspectiva comparada desde el año 1999 a 2008.

Palabras clave:

Ciberdemocracia, comunicación política, Internet parlamentario, correo electrónico, Congreso de los Diputados

Abstract:

The new information technologies, and specifically the Internet, have spread the idea that politicians and citizens can communicate directly using some of the most popular tools, such as e-mail. This article summarizes the main results of an investigation aimed to ascertain the response rate may have a common citizen to send an email to members of Congress in a comparative perspective from 1999 to 2008.

Key Words:

Cyberdemocracy, Political Communication, Parliamentary Internet, E-mail, Chamber of Deputies 


\section{Introducción}

Internet ha incrementado en la sociedad de la información las posibilidades de comunicación entre ciudadanos y políticos. Entre las diferentes herramientas que ambos agentes utilizan figuran aquellas que permiten únicamente obtener información por parte de quién la busca, y las herramientas que añaden la posibilidad de interacción, entendiendo la diferencia entre interactividad e interacción como la describe Frau-Meigs (2001) de modo que mientras la primera de ellas comprende la comunicación o diálogo entre persona-máquina, la interacción implica un diálogo entre personas con mediación de una máquina.

En el ámbito de la interacción entre representantes y representados el correo electrónico es, sin duda, una de la herramienta que despierta más esperanzas (Chappelet, 2004; Tucker, 2004; Chen, 2004; Carter, 1999, entre otros), bien sea porque se trate de la modalidad más utilizada (Frick, 2005) o una de las herramientas más populares ${ }^{1}$, bien por su capacidad para suplir los canales convencionales de correspondencia (Norris, 2001).

Es preciso enmarcar el correo electrónico dentro del grupo de herramientas de la Web 1.0 (Sánchez González, 2010 y López García, 2005) junto a los formularios, encuestas, chats, foros de debate, tablones de anuncios, entre otros, y reservar para la llamada Web 2.0, los blogs, wikis o redes sociales (López, 2008). A fecha de junio de 2010, y teniendo en cuenta la presencia o ausencia de estas herramientas, la web institucional del Congreso de los Diputados todavía no había dado el salto a la Web 2.0.

Aplicado al ámbito de la participación ciudadana en Internet, el e-mail se enmarca dentro de las herramientas de interpelación política de la ciberdemocracia (para una definición detallada del término véase Campos, 2009). La democracia interpelativa, planteada por Dader (2001), supone un escalón más modesto de la democracia deliberativa en la que los ciudadanos disponen de recursos eficaces para interpelar a sus representantes, obteniendo por su parte algún tipo de respuesta. Se sitúa, no obstante, en un estadio superior al proceso de rendición de cuentas en cuanto no conlleva únicamente una fiscalización de la información sobre la base de la documentación transparente, sino también una ampliación de la información sobre la base de documentación transparente de la que los ciudadanos ya disponen.

Por tanto, el correo electrónico, como herramienta de interpelación política, permite al ciudadano contactar con sus gobernantes o representantes para solicitarles e inquirirles información o respuestas específicas a preguntas y comentarios. En ciertas circunstancias la interpelación puede dar lugar a un estadio cualita-

Según la última Encuesta sobre Equipamiento y Uso de Tecnologías de la Información y Comunicación en los hogares (INE, 2009), el 86,2\% de los usuarios de Internet en España utiliza los servicios de comunicación en la red para recibir o enviar correos electrónicos. 
tivo superior en la ciberdemocracia si el intercambio inicial permite acceder a algún tipo de diálogo más amplio entre el ciudadano y el representante político. Tal opción, obviamente, dependerá de que el político o gobernante interpelado acceda a mantener, mediante correo electrónico o una vía de interacción complementaria, un intercambio de impresiones más profundo que la escueta respuesta de datos o de declaraciones.

\section{El correo electrónico como herramienta de la democracia interpelativa}

Como fase previa, el gobernante ha de facilitar una dirección de correo electrónico a los ciudadanos en un sitio de acceso público. En esta línea, y tomando como referencia el Congreso de los Diputados, a finales de la VIII Legislatura de las Cortes Generales el 86,3\% de los parlamentarios ofrecía un $e$-mail en el sitio web de la Cámara Baja y únicamente 48 de los 350 diputados no lo publicaban (tabla 1).

Como punto de partida, cabe indicar que la investigación se fundamenta en un experimento que toma como modelo el trabajo realizado previamente por Dader (2003), y Dader y Campos (2006), basado en el envío de mensajes electrónicos a todos los diputados con una dirección de correo electrónico pública en el sitio web de la Cámara Baja para conocer cuántos de ellos responden a un mensaje enviado por un supuesto ciudadano común.

Tabla 1. Población de estudio: diputados con e-mail público a principios de 2008

\begin{tabular}{|lcc|}
\hline & \multicolumn{2}{c|}{$\begin{array}{c}\text { Muestra teórica } \\
\text { (diputados con } e \text {-mail público) }\end{array}$} \\
\hline Con correo electrónico institucional & Datos absolutos & Datos relativos \\
\hline Con correo electrónico institucional y no-institucional & 261 & 86,4 \\
\hline Con correo electrónico no-institucional $\left(^{*}\right)$ & 39 & 12,9 \\
\hline Total de diputados con $e$-mail público & 2 & 0,7 \\
\hline Sin correo electrónico público & 302 & 86,3 \\
\hline Total de diputados & 48 & 13,7 \\
\hline
\end{tabular}

Nota $\left({ }^{*}\right)$ : El correo electrónico no-institucional es el ajeno al ofrecido por el Congreso (@diputado.congreso.es) en algunos casos es una dirección de correo privada de un servidor gratuito y en otros la cuenta de correo del partido político.

Fuente: Elaboración propia basada en la información recogida en www.congreso.es, 2008 
Con todo ello, este artículo pretende ofrecer datos pertinentes a las siguientes cuestiones:

Cuántos de los parlamentarios que componían el pleno del Congreso de los Diputados en la VIII Legislatura (2004-2008) y publicaban su dirección de $e$-mail respondían a un correo electrónico remitido simulando ser un ciudadano de a pie.

Tomando como referencia los resultados de los ensayos previos realizados desde el año 1999, conocer en qué número había aumentado, o disminuido, el total de diputados que voluntariamente hacían pública su dirección de $e$-mail y de los que respondían al correo electrónico desde la primera medición realizada por Dader (2003).

Averiguar si en campaña electoral los diputados respondían al correo electrónico más que en otros períodos oficialmente no electorales y si, en la misma línea, los parlamentarios utilizaban esta vía para pedir el voto para su partido.

Como hipótesis de partida, se considera, en primer lugar, que los representantes de la Cámara Baja en la VIII Legislatura responden en mayor número a los correos electrónicos de los ciudadanos en campaña electoral que en períodos no electorales. En segundo lugar, se predice que, aun así, el índice de respuesta que los ciudadanos pueden obtener de los parlamentarios por correo electrónico es muy minoritario. Finalmente, se pronostica que en escasos casos la respuesta proviene de políticos de primera fila.

Para llevar a cabo este experimento, la autora empleó cuatro cuentas de correo electrónico creadas ad hoc en dos servidores distintos gratuitos de Internet para enviar un mensaje, el día 1 de marzo de 2008, a cada parlamentario con un e-mail público. El contenido del mensaje toma como referencia el texto que José Luis Dader (2003) remitió con anterioridad a los diputados con ligeras variaciones relativas a la petición del programa electoral del partido político al que pertenecían -en lugar de solicitar información sobre la posibilidad de conseguir una invitación a la sede del Congreso de los Diputados, como solicitaba dicho autor ${ }^{2}$ - con el fin de realizar un análisis comparativo de los datos resultantes en las distintas mediciones.

2 El mensaje remitido a los diputados fue el siguiente: "Estimado[a] señor[a] [nombre del diputado/a]: Soy nuevo en esto de Internet y entrando en las páginas web del Congreso me he encontrado con que ustedes los diputados ofrecen una dirección de correo electrónico al parecer abierta a todo el mundo. Mi curiosidad es saber si esta dirección sirve para que la gente normal y corriente como yo pueda ponerse en contacto con ustedes, o si por el contrario reservan esta vía para las comunicaciones con políticos, instituciones y medios de comunicación. Por cercanía hacia su grupo político he elegido su dirección en lugar de otras y si fuera tan amable de responderme me gustaría saber también si puede facilitarme el programa electoral del [nombre del partido político] o indicarme a quién o a dónde he de dirigirme para conseguirlo". 
Este tipo de experimento, con utilización de una ocultación o engaño para conseguir que el sujeto sometido a experimentación no detecte que el supuesto ciudadano común es en realidad una investigadora, suscita en muchas personas, e incluso en algunos ambientes académicos, ciertas dudas deontológicas que también han sido sometidas a reflexión y contraste. Siguiendo a Wimmer y Dominick (1996: 421 y ss.), a partir de lo aportado por diversos especialistas en deontología de la investigación psicosocial (Elms, 1982 y otros), la ocultación de cierta información a los participantes en un experimento u observación psicosociológica resulta plenamente legitimada en términos éticos y está avalada por los comités de deontología científica más exigentes siempre que se den ciertas circunstancias como, entre otras, que no existan otros procedimientos para obtener la información científica buscada -en este caso, los sujetos estudiados debían ser observados en su comportamiento natural, el cual quedaría modificado en el momento mismo de saber que forman parte de un estudio-, que sea mínima la gravedad de la ocultación o engaño planteado y que éste no afecte básicamente a la intimidad o privacidad del sujeto analizado.

\section{Resultados de una comparación histórica (1999-2008): el uso del e-mail por parte de los diputados como vía de comunicación con los ciudadanos}

El número de diputados con, al menos, una dirección de correo electrónico aumentó progresivamente desde el año 1999 hasta 2008 (gráfico 1). No obstante, en el transcurso de la última legislatura (2004-2008) sólo un diputado más publicó su buzón electrónico. Este dato revela que, por un lado, durante el período de una misma legislatura el número de diputados que publicaban su correo permaneció prácticamente invariable y, por otro, que fue precisamente en el cambio de legislatura cuando se produjo una renovación de intenciones: bien algunos diputados que conservaban su acta decidieron publicar su cuenta de correo sin haberlo hecho antes, o bien los parlamentarios que estrenaron escaño se mostraron más predispuestos e interesados en publicar su correo. 
Gráfico 1. Diputados que responden al correo electrónico (1999-2008)

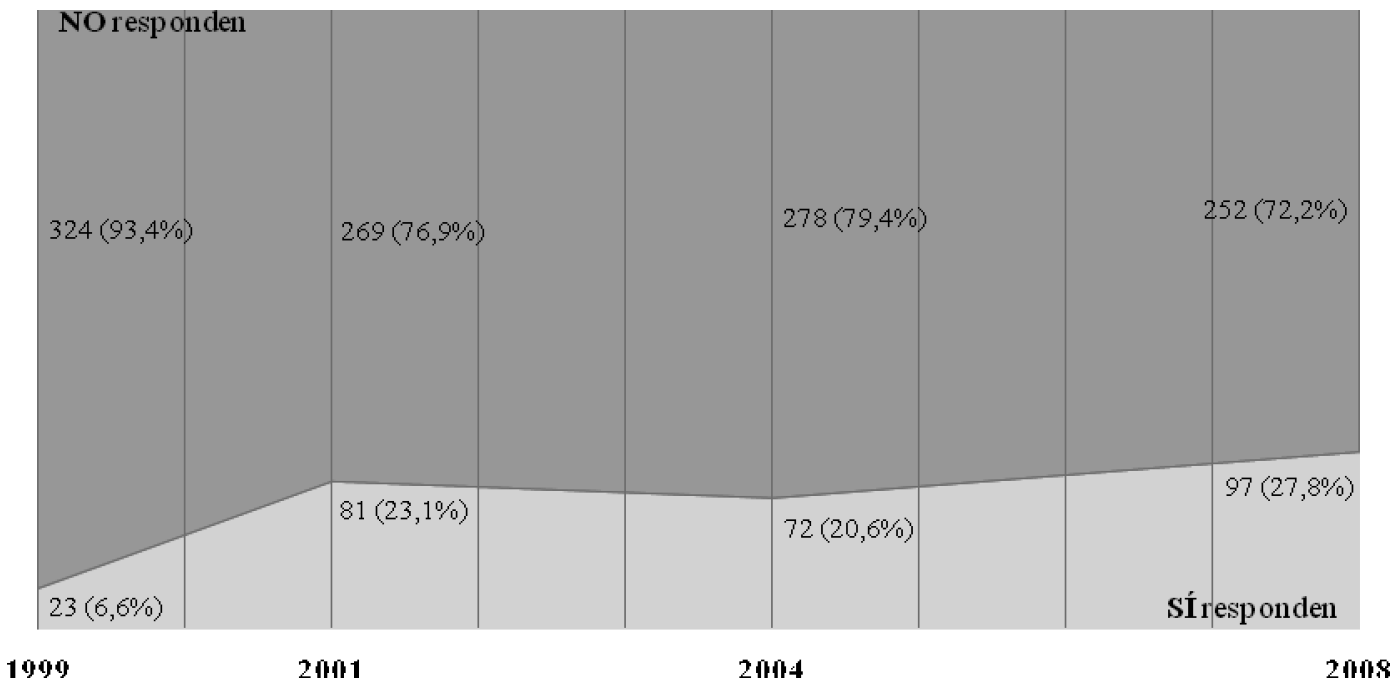

NOTA: En gris claro los diputados que responden al e-mail en datos absolutos y en $\%$ sobre el total de diputados del

Congreso. En gris oscuro diputados que no responden al correo electrónico en datos absolutos y en \% sobre el total de diputados.

Es posible que en la VIII Legislatura se haya llegado prácticamente al tope de diputados que deseen publicar su correo (de los 350, 302 lo hacían), y que parlamentarios que desde los primeros ensayos de este experimento venían sin publicar su correo, no lo vayan a hacer con el avance de las legislaturas, y exista, por tanto, un margen en torno al $10 \%$ de diputados que, al menos a corto plazo, no vayan a ofrecer su dirección de $e$-mail en el sitio web del Congreso. Así, también puede ocurrir que representantes que publicaban su correo no lo hagan en las siguientes legislaturas -como ocurre con algunos de los parlamentarios que en la VIII ofrecían su e-mail y a principios de la IX ya no lo hacían- de tal modo que el número de nuevos diputados que publiquen su dirección electrónica se vaya compensando con la de los otros que no lo hacen.

A propósito del número de diputados que publicaban su correo electrónico en 2008, y tomando como referencia los resultados de 1999, 2001 y 2004-05, recogidos en Dader (2003) y Dader y Campos (2006), pueden distinguirse las siguientes tendencias al compararlos con los resultados del ensayo realizado en la última legislatura: 
Por grupos parlamentarios: atendiendo a los mayoritarios, los diputados del PSOE publicaban en mayor número su cuenta de correo que los del PP, y esta tendencia se mantuvo desde el primer ensayo, en 1999: el 37,5\% de los parlamentarios socialistas ofrecían su e-mail ese año y del PP lo hicieron el 15,4\%. En 2001 el 73,6\% de los representantes del PSOE publicaban su correo frente al 52,7\% de los populares. En la VIII Legislatura -con el Partido Popular en la oposición- el 92\% de los parlamentarios socialistas facilitaban su buzón electrónico en 2004-05 y el 92,7\% en 2008, frente al 80,5\% de los populares en 2004-05 y al 80,3\% al término de la referida legislatura. En cuanto al resto de los grupos parlamentarios, en la VIII Legislatura continuaron su tendencia de mejora mencionada por Dader y Campos (2006), con una reseña negativa al PNV que seguía siendo el grupo parlamentario más opaco en cuanto a la difusión de las direcciones de correo electrónico de sus diputados. Por lo que se refiere a Izquierda Unida, se engloba en la tendencia de mejora aludida, ya que pasó de contar con 3 diputados con e-mail público en 1999, con 4 a principios de la VIII (2004-05) y 5 a finales de la misma; es decir: en 2008 todos los diputados de IU-ICV publicaban su correo electrónico. Esta misma tendencia era compartida por CiU que desde 1999 fue incrementando el número de diputados con $e$-mail hasta alcanzar el 100\% en la VIII Legislatura.

Por sexo: ambos, hombres y mujeres, han ido incrementado su número, pero con un avance más rápido entre las mujeres que entre los varones. En 1999 el porcentaje de diputadas con cuenta pública era inferior al de sus colegas varones -el 17,8\% frente al 28\% de los hombres-, en 2001 el número de parlamentarias que facilitaban su e-mail superó al de los parlamentarios -el 65,7\% de ellas, frente al 56,4\% de ellos- y esta tendencia se consolidó en la VIII Legislatura donde el porcentaje de diputadas predispuestas a publicar su buzón electrónico siguió creciendo mientras que el de diputados decreció ligeramente: el 88,4\% en 200405 y el 89,9\% en 2008, por lo que respecta a las diputadas, frente al 84,6\% de los varones en 2004-05 y el $84,2 \%$ en 2008.

Al correo electrónico remitido a los $301+{ }^{3}$ diputados con una dirección electrónica pública, en mayo de 2008, respondieron un total de 97, lo que supone que a finales de la VIII Legislatura el 27,8\% de los parlamentarios del Congreso respondían a los mensajes remitidos vía $e$-mail por ciudadanos comunes.

\footnotetext{
3 Señalizado con (+) los datos en los que se excluye al diputado popular Rogelio Baón, que falleció en febrero de 2008, cuando ya se había medido el número de diputados con e-mail público pero, lógicamente, no se computa en la medición de diputados que responden, o no, al correo electrónico. Por tanto, incluyendo al referido diputado los parlamentarios con e-mail público eran 302, y sin computar al mismo, 301+.
} 
Tabla 2. Evolución de respuestas al $e$-mail entre la VI y la VIII Legislatura parlamentaria de las Cortes Generales (1999-2008)

\begin{tabular}{|lcccccccc|}
\hline Diputados & \multicolumn{2}{c}{ VI Legislatura } & \multicolumn{2}{c}{ VII Legislatura } & \multicolumn{3}{c|}{ VIII Legislatura } \\
\hline & & 1999 & \multicolumn{2}{c}{2001} & \multicolumn{2}{c}{2004} & 2008 \\
\hline & $\mathrm{R}$ & $\%$ & $\mathrm{R}$ & $\%$ & $\mathrm{R}$ & $\%$ & $\mathrm{R}$ & $\%$ \\
\hline CON $e$-mail & 92 & 26,5 & 210 & 60 & 301 & 86 & $301^{+}$ & 86,3 \\
\hline SÍ responden & 23 & 6,6 & 81 & 23,1 & 72 & 20,6 & 97 & 27,8 \\
\hline NO responden & 324 & 93,4 & 269 & 76,9 & 278 & 79,4 & 252 & 72,2 \\
\hline Total & $347^{1}$ & 100 & 350 & 100 & 350 & 100 & $349^{+}$ & 100 \\
\hline
\end{tabular}

NOTA: R Total de diputados con e-mail o diputados que responden al correo \% sobre total de diputados. Los datos de los años 1999, 2001 y 2004 corresponden a Dader y Campos (2006) (1) Excluidos los 3 diputados de Herri Batasuna. (+) Excluido el diputado popular Rogelio Baón, que falleció en febrero de 2008.

Este porcentaje de respuesta obtenido en la última medición es superior al logrado en cualquier otro ensayo previo (tabla 2). No obstante, no es posible concluir que en período electoral (en este caso, mayo de 2008) los diputados respondan en mayor número a los mensajes ciudadanos que en otros momentos de la legislatura. Más bien, los datos apuntan a la necesidad de realizar nuevos ensayos que permitan nuevas muestras en ambos períodos para un análisis comparativo.

\section{Perfil de los diputados que responden al $e$-mail de un supuesto ciudadano a finales de la VIII Le- gislatura}

Para tratar de establecer un perfil de los diputados que contestan al correo electrónico se observan los datos atendiendo a las siguientes variables: grupo político, tipo de parlamentario, de cuenta de correo, antigüedad en el Congreso, sexo, edad, nivel de estudios, y, finalmente, circunscripción del representante.

\subsection{Por grupo político: los diputados socialistas responden en mayor número al correo electrónico que los populares}

Considerando, en primer lugar, a los grupos mayoritarios, se aprecia una mayor predisposición entre los parlamentarios del Grupo Parlamentario Socialista que entre los representantes del Grupo Popular en el 
Congreso tanto a la hora de publicitar las direcciones de correo electrónico como a la de responder a los mensajes (gráfico 2).

Gráfico 2. Diputados que responden al e-mail por grupo parlamentario

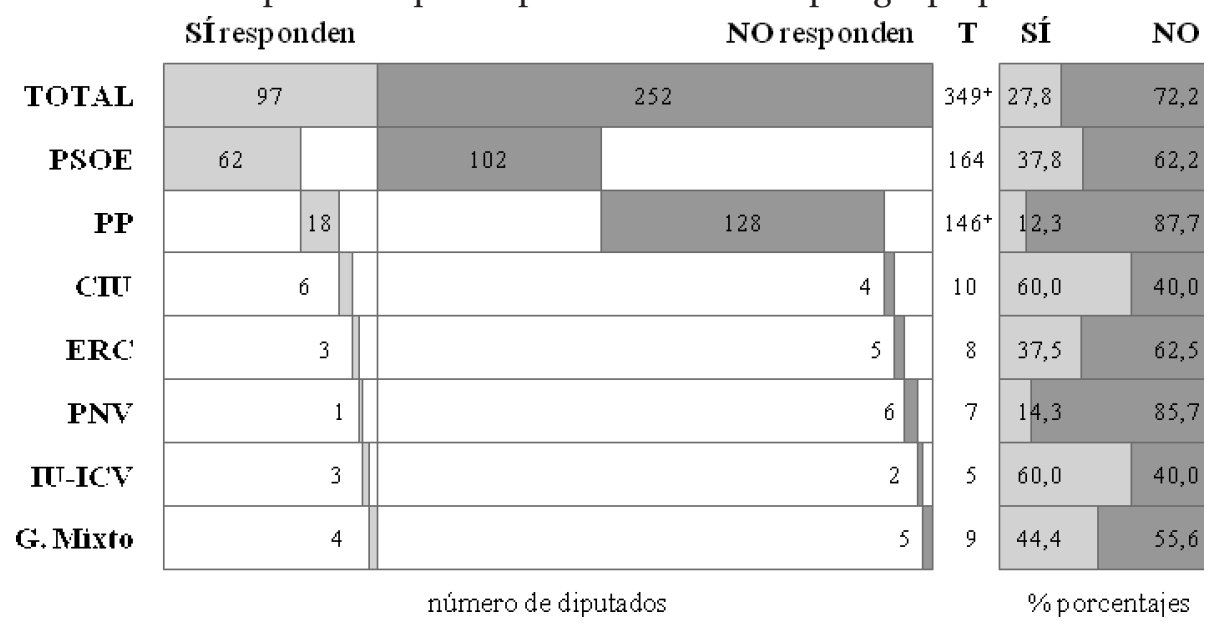

NOTA: Sombreado en gris claro diputados que responden y en oscuro, que no responden. T Total de diputados en el Congreso por grupo parlamentario \% de diputados con y sin respuesta sobre T + Excluido el diputado del PP Rogelio Baón, que falleció durante la campaña electoral de 2008.

En cuanto al resto de los grupos parlamentarios, la tendencia de estos diputados es muy variable entre ellos: frente a IU -con publicación de dirección electrónica por parte de todos sus diputados en 2008 y un índice de respuesta del 60\% - y a CiU -con todos los diputados con $e$-mail público en la misma fecha y el mismo índice de respuesta al mensaje enviado que IU- se sitúan el PNV y ERC con datos más modestos, principalmente del Partido Nacionalista Vasco, tanto a publicar las cuentas de correo electrónico como a responder a los mensajes recibidos.

En cuanto a los partidos que conforman el Grupo Mixto, la tendencia de este colectivo tanto a facilitar su cuenta de correo como a responder a los mensajes, depende del político que ocupe el acta de diputado: frente a unos comprometidos como José Antonio Labordeta (CHA) o Begoña Lasagabaster (EA), se sitúan Francisco Rodríguez (BNG) o Uxue Barkos (Na-Bai) que no respondieron al mensaje remitido. 


\subsection{Por tipo de diputado: los parlamentarios de segunda línea responden más que las élites políticas}

En un primer grupo se clasifican los diputados de élite pasivos o silenciosos ante la posibilidad de responder al ciudadano de a pie, en este experimento. En dicho grupo se aglutinan tanto los candidatos líderes de la lista principal -entre ellos, José Luis Rodríguez (PSOE) o Mariano Rajoy (PP), que ni siquiera llegó a publicar su dirección de correo- como los portavoces de los dos grandes grupos parlamentarios -Diego López Garrido (PSOE) que no respondió, y Eduardo Zaplana (PP), que ni siquiera ofrecía su e-mail-. Estos máximos representantes no respondieron ni al mensaje enviado en $2008 \mathrm{ni}$ a los anteriores.

En un segundo grupo figuran los diputados que, siendo candidatos líderes de la lista principal de su partido en 2004 fueron, también, portavoces de su grupo parlamentario durante la VIII Legislatura. La tendencia de estos parlamentarios fue positiva al responder, en su mayoría, al mensaje enviado: Josep Antoni Durán i Lleida (CiU), Joan Herrera (ICV), José Antonio Labordeta (CHA) y Begoña Lasagabaster (EA), así lo hicieron. Por su parte, Ana María Oramas, -aunque no había sido candidata líder de su partido en 2004, sí lo fue en 2008, así como portavoz de su grupo desde 2007-, también respondió al mensaje. En contraposición, los parlamentarios que fueron candidatos líderes y portavoces de IU, PNV, Na-Bai, BNG, no contestaron ninguno al mensaje enviado.

En un tercer grupo se aglutinan los parlamentarios de segunda línea -aquellos que no fueron ni candidatos líderes de la lista principal de su partido en 2004, ni portavoces de su grupo parlamentario en la VIII Legislatura-. De los $235^{+}$parlamentarios de este grupo contestaron una minoría -un total de 92, el 27,5\% del total de parlamentarios de la cámara-, entre los que destacan los diputados de CiU -de los 9 respondieron 5- y los de IU-ICV -de los 3 representantes respondieron 2-. Por lo que se refiere a los diputados del PSOE y del PP de segunda fila, de los 162 socialistas respondieron 62 (el 38,3\%) y de los $144^{+}$populares contestaron únicamente 18 (el 12,5\%).

Finalmente, dentro de este tercer grupo de diputados de segunda línea cabe hablar de los parlamentarios que, sin ser portavoces del grupo parlamentario ni candidatos líderes, y aún formando parte de los denominados diputados de segunda línea, son políticos conocidos (tanto en la VIII Legislatura, como en otras anteriores).

\subsection{Por tipo de e-mail: los representantes que ofrecen más de una dirección de correo responden en mayor número al mensaje ciudadano}

Los diputados que a finales de la VIII Legislatura ofrecían dos direcciones de correo electrónico en el sitio web del Congreso (tabla 1) fueron los más voluntariosos a la hora de responder el e-mail enviado: de los 39 
parlamentarios que anunciaron dos direcciones electrónicas, el 56,4\% respondió al correo remitido (un total de 22). Seguidos de los parlamentarios con el correo electrónico institucional: de los $260^{+}$contestó el $28,8 \%$ (un total de 75 ). Finalmente, los dos dirigentes que ofrecían su e-mail privado, no respondieron al mensaje electrónico.

\subsection{Por antigüedad en la Cámara: los diputados noveles en el Congreso contestan más al e-mail}

Los diputados que en la VIII Legislatura ocupaban su escaño por primera vez fueron los más propensos a facilitar su e-mail y, también, a contestar al mensaje electrónico remitido, seguidos de los parlamentarios que lo eran por cuarta vez. A estos les seguían los representantes que contaban con más antigüedad en la Cámara Baja (en la VIII ocupaban su acta de diputado en su quinta o más legislatura). Los más reacios fueron los parlamentarios que ocupaban su escaño en su tercera legislatura, seguidos de los que eran diputados por segunda vez (gráfico 3).

Gráfico 3. Diputados que responden al $e$-mail por antigüedad en el Congreso

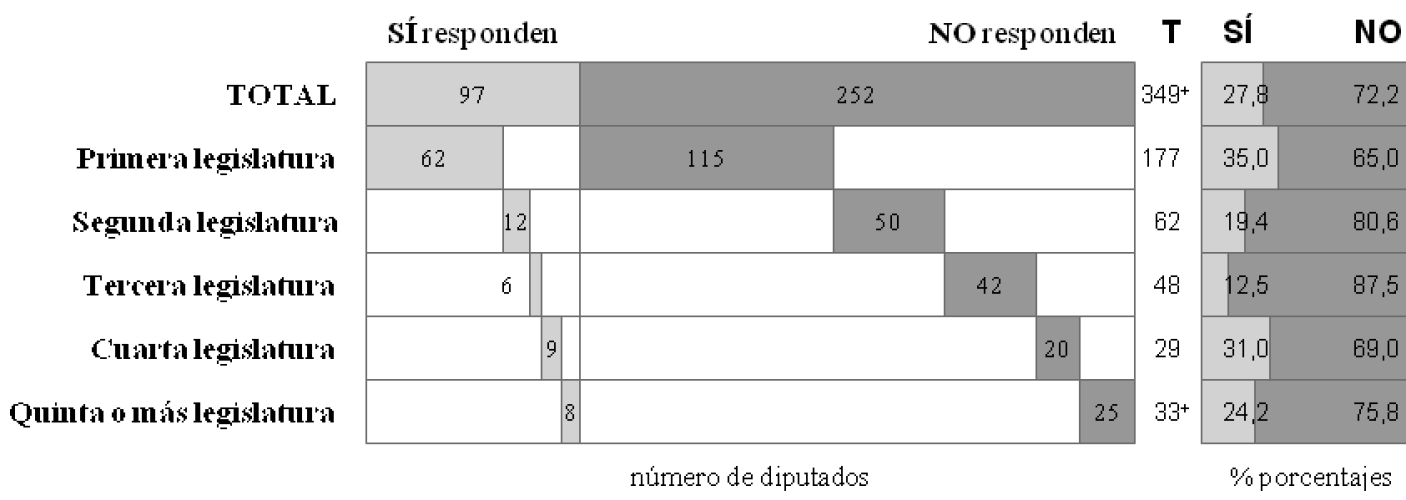

NOTA: Sombreado en gris claro diputados que responden y en oscuro, que no responden. T Total de diputados en el Congreso por antigüedad \% de diputados con y sin respuesta sobre T + Excluido el diputado del PP Rogelio Baón, que falleció durante la campaña electoral de 2008.

\subsection{Por sexo: las diputadas responden más que los diputados}

Las mujeres mostraron una mayor predisposición que sus colegas varones tanto a publicar su e-mail como a responder al correo electrónico remitido en 2008. Por tanto, a finales de la VIII Legislatura se afianza la ten- 
dencia iniciada en 2001 (Dader 2003 y Dader y Campos 2006) de una ligera superioridad de las diputadas sobre los diputados a la hora de publicar y responder al correo electrónico (tabla 3).

Tabla 3. Diputados que responden en la VIII Legislatura al $e$-mail por sexo

\begin{tabular}{|lccccccc|}
\hline & $\begin{array}{c}\text { Total D. } \\
\text { en Congreso }\end{array}$ & & 2004-05 & & \multicolumn{3}{c|}{ 2008 } \\
\hline & & $e$-mail & $\mathrm{R}$ & $\%$ & $e$-mail & $\mathrm{R}$ & $\%$ \\
\hline Diputados & $221^{*}$ & 187 & 42 & 19,0 & $187^{+}$ & 56 & $25,4^{+}$ \\
\hline Diputadas & 129 & 114 & 30 & 23,3 & 116 & 41 & 31,8 \\
\hline Total & $\mathbf{3 5 0 *}$ & $\mathbf{3 0 1}$ & $\mathbf{7 2}$ & $\mathbf{2 0 , 6}$ & $\mathbf{3 0 1}^{+}$ & $\mathbf{9 7}$ & $\mathbf{2 7 , 8 ^ { + }}$ \\
\hline
\end{tabular}

NOTA: $\mathbf{R}$ Total de respuestas por grupo parlamentario \% Porcentaje de respuestas por diputados sobre el total de cada categoría. (*) En el envío de 2008 se calcula sobre 220+ y 349+, al excluir al diputado Baón.Datos de 2004-05 corresponden a Dader y Campos (2006).

4.6. Por edad: los parlamentarios nacidos entre 1976 y 1967, los más implicados con las respuestas a los ciudadanos

Si en las mediciones anteriores realizadas por Dader (2003) y Dader y Campos (2006) se venía observando una tendencia general de: a menor edad del diputado mayor tasa de respuesta al $e$-mail, lo cierto es que los resultados de 2008 muestran que si bien los parlamentarios más jóvenes -entre 20 y 30 años-siguen siendo, porcentualmente, los más propensos a facilitar su buzón electrónico, ya no son los representantes con mayor índice de respuesta.

En este ensayo, los representantes con menos edad respondieron en igual número que los del grupo de edad máxima -más de 60 años-, mientras que el pico de repuesta más alto se alcanzó entre los parlamentarios de 31 a 40 años -nacidos entre 1976 y 1967- que, precisamente, coincidían con los diputados más jóvenes en los experimentos previos llevados a cabo entre 1999 y 2004 (gráfico 4). Es decir, los datos señalan la existencia de una generación de diputados especialmente comprometida con el uso del correo electrónico, que mantiene su mayor disponibilidad electrónica según avanzan las legislaturas y que, por lo tanto, implica un paso al tramo generacional siguiente en cada nueva legislatura. 
Gráfico 4. Diputados que responden al $e$-mail por edad

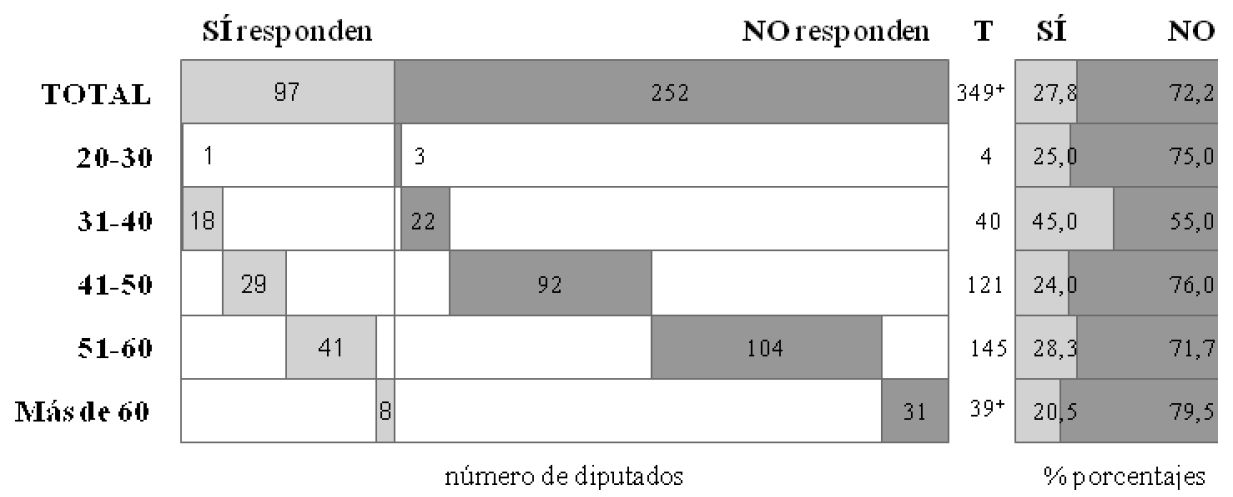

NOTA: Sombreado en gris claro diputados que responden y en oscuro, que no responden. T Total de diputados en el Congreso por grupos generacionales \% de diputados con y sin respuesta sobre $\mathrm{T}+$ Excluido el diputado del PP Rogelio Baón, que falleció durante la campaña electoral de 2008.

4.7. Por nivel de estudios: a mayor nivel de formación de los diputados, mayor índice de respuesta a los ciudadanos

En cuanto al nivel de estudios, se distinguen dos tendencias diferentes: la primera de ellas referida al número de parlamentarios que ofrecía su e-mail en el sitio web del Congreso de los diputados; y la segunda, en relación al índice de respuesta de estos representantes al mensaje electrónico enviado. 
Gráfico 5. Diputados que responden al e-mail por nivel de estudios

\begin{tabular}{|c|c|c|c|c|c|c|}
\hline & sÍresponden & & NO resp onden & $\mathbf{T}$ & sí & No \\
\hline TOTAL & 97 & 252 & & $349+$ & 27,8 & 72,2 \\
\hline Primarios & 0 & 1 & & 1 & 0,0 & 100,0 \\
\hline Medios & 3 & 10 & & 13 & 23,1 & 76,9 \\
\hline Tniversit, imacabados & 7 & 13 & & 20 & 35,0 & 65,0 \\
\hline Tniversitarios finalizados & 54 & 146 & & $200^{+}$ & 27,0 & 73,0 \\
\hline Posgrado & 12 & & 31 & 43 & 27,9 & 72,1 \\
\hline Doctorado & 11 & & 24 & 35 & 31,4 & 68,6 \\
\hline Otros & 3 & & 13 & 16 & 18,8 & 81,3 \\
\hline Sin dato & 7 & & 14 & 21 & 33,3 & 66,7 \\
\hline
\end{tabular}

NOTA: Sombreado en gris claro diputados que responden y en oscuro, que no responden. T Total de diputados en el Congreso por nivel de estudios \% de diputados con y sin respuesta sobre T + Excluido el diputado del PP Rogelio Baón, que falleció durante la campaña electoral de 2008.

En el primer caso -sin tener en cuenta al único parlamentario con estudios primarios, que no publicó su correo electrónico-, los representantes con estudios medios y universitarios inacabados fueron más proclives a facilitar su e-mail que los diputados con estudios universitarios y posteriores.

En cambio, en las respuestas al mensaje remitido, los parlamentarios con estudios universitarios inacabados fueron los más propensos a responder (el 35\%), seguidos de los diputados con grado de doctor $(31,4 \%)$, estudios de posgrado $(27,9 \%)$ y universitarios finalizados (27\%). De los diputados con estudios medios, respondió el 23,1\%. En términos generales, sí se aprecia una mayor predisposición de respuestas en los diputados con estudios universitarios y estudios superiores al grado, que los parlamentarios con estudios medios.

\subsection{Por circunscripciones: los diputados catalanes responden en mayor número al mensaje electrónico}

Atendiendo al índice de respuestas por circunscripciones, tal y como venía sucediendo en las experiencias previas de 1999 a 2004 (Dader y Campos, 2006), Cataluña (con un índice de respuesta del 50\% sobre el total 
de sus diputados) seguía siendo a finales de la VIII Legislatura una de las comunidades donde sus parlamentarios en el Congreso respondían en mayor número al correo electrónico, si bien en el ensayo de 2008 las comunidades de Aragón y Baleares -que en experimentos previos se situaban en los primeros puestos del ranking junto a Cataluña-registraron un menor índice de respuestas, y ocupaban sus puestos Cantabria -el 60\% de sus diputados respondieron al mensaje- y La Rioja (50\%).

Por el contrario, al margen de Ceuta y Melilla-donde su único diputado en el Congreso no respondió en ningún caso al mensaje remitido- Extremadura, Galicia y Castilla-La Mancha fueron las comunidades con menor índice de respuestas -respectivamente: 0\%, 8,3\% y 15\% de diputados que respondieron al correo electrónico-. Madrid y Asturias -que en ensayos previos se situaban, junto a Galicia, entre los puestos más bajos de respuesta- registraron una pequeña mejoría en el último experimento con tasas de respuesta del $17,6 \%$ y del 25\%, respectivamente. A pesar de esta ligera mejoría, Madrid obtuvo un índice de respuesta muy bajo, sobre todo si se compara con la comunidad de Cataluña o con la provincia de Barcelona, como circunscripciones más análogas en volumen de representantes.

Detalladamente, por provincias, los diputados de Cantabria, Gerona y Santa Cruz de Tenerife alcanzaron tasas de respuesta superiores al 50\%, mientras que ninguno de los parlamentarios de Zamora, Valladolid, Salamanca, Ourense, Melilla, Lugo, Guadalajara, Cuenca, Ceuta, Cáceres, Badajoz, Álava y La Coruña respondió al mensaje electrónico.

En los extremos, y en referencia a los ensayos previos citados anteriormente, Gerona se consolidaba en 2008 como una de las provincias que desde los primeros experimentos venía mostrando una tasa de compromiso más alta que el resto de las provincias. Mientras que Orense, Salamanca, Segovia, Zamora y Ávila seguían conservando los puestos más bajos en la escala de respuestas.

\section{Consideraciones adicionales sobre el contenido y forma del mensaje de respuesta de los diputados}

La mayoría de los diputados respondieron, en mayo de 2008, al e-mail enviado desde su cuenta de correo institucional del Congreso (73 de los 97). Un total de quince enviaron el mensaje de respuesta desde el buzón de una institución distinta a la Cámara Baja -generalmente de una institución pública con la que el diputado estaba vinculado o desde el su partido político-, mientras que los ocho diputados restantes respondieron desde una cuenta de correo personal -todos ellos, desde un servidor de correo gratuito-.

Ciertamente, no es posible conocer quién fue el autor real de los mensajes de respuesta: si el propio diputado o un asistente. No obstante, la mayoría de las respuestas recibidas (89 de las 97) estaban firmadas por 
el propio parlamentario, y el resto por otra persona que escribía en nombre del parlamentario y firmaba como su asistente o ayudante.

En general, por las respuestas obtenidas se deduce que la mayoría de los diputados gestionaban ellos mismos su cuenta de correo electrónico. Así, una diputada socialista afirmaba: "Efectivamente este es mi correo personal y, en mi caso, lo veo y lo contesto yo misma en exclusiva", y otro diputado de IU-ICV indicaba: "Hay diputados que la tienen [la dirección de correo electrónico] desviada al ordenador de los asistentes de la sede, pero yo prefiero administrarla personalmente".

El mensaje remitido a todos los diputados con un e-mail público fue enviado el día 1 de marzo de 2008 que, intencionadamente, coincidía en sábado. Sobresale el hecho de que 19 de los 97 parlamentarios que contestaron lo hicieron el mismo día, e incluso a las pocas horas de haberlo remitido. Al día siguiente, domingo, respondieron 25 diputados más. El lunes, primer día laborable desde el envío del mensaje, contestaron 28 parlamentarios. Ningún diputado respondió ni durante el día de reflexión (el 8 de marzo) ni tampoco durante la jornada electoral. Pasada esta fecha contestaron ocho diputados más, y la más tardía de todas las respuestas fue recibida un mes después de haber remitido el mensaje.

Aunque el contenido de los mensajes enviados por los diputados como respuesta fue cordial, cabe indicar que solo 19 de los 97 diputados manifestaron expresamente su disponibilidad para continuar la comunicación con el ciudadano, si este lo consideraba oportuno, a través de un nuevo correo electrónico o por vía telefónica -para lo que algunos facilitaban su número de teléfono móvil personal-. Es destacable que algunos de los diputados que en las elecciones de 2008 no se presentaban a la renovación de su acta de diputados ofrecían, sin embargo, sus datos de contacto para esclarecer dudas o completar información. No obstante, en contra de lo descrito en Dader y Campos (2006), en esta ocasión, aunque algunos de los parlamentarios facilitaban sus números de teléfono, ninguno de ellos aludió a la posibilidad de concretar entrevistas personales en la sede parlamentaria o en la de la circunscripción local de su partido.

Algunos de los diputados que respondieron al mensaje remitido añadieron otro tipo de información no solicitada explícitamente en el $e$-mail pero que revelaba notas útiles sobre el tipo de mensajes que reciben los diputados de los ciudadanos, su hábito de respuesta a estos correos y la repercusión que tenían estos mensajes en su actividad parlamentaria.

En esta línea, las respuestas recibidas de los parlamentarios mostraron que el volumen de mensajes que los ciudadanos escriben es variable, en función del diputado. Así, frente al diputado del GS que indicaba que: "Desgraciadamente, en contra de lo que yo creía, no hay demasiadas consultas serias por este medio (nor- 
malmente recibo cientos de mensajes iguales de colectivos que protestan por algo, pero eso tampoco es una comunicación directa con el ciudadano, sino una forma de recibir protestas)", se localiza la diputada del Grupo Mixto que precisaba: "Ciertamente a lo largo de los años que he desarrollado el trabajo de diputada (obviamente más en los últimos tiempos por cuestiones de mejora de tecnología y apertura de la misma a un mayor número de personas), han sido muchísimas las personas que se han puesto en contacto conmigo para solicitar información o expresar sus opiniones".

El hábito de respuesta mostrado por estos diputados a los mensajes ciudadanos varía entre aquellos parlamentarios que afirmaron responder, o al menos intentarlo, a todos los correos de ciudadanos -por ejemplo, un diputado del Grupo Popular afirmaba: "Siempre haré lo posible por contestarte, aunque a veces nos inundan con miles de correos y nos dificultan nuestra labor"-, los parlamentarios que abiertamente declaraban que solo responden a los ciudadanos de su circunscripción o a los que les escriben sobre temas vinculados a su labor en la Cámara Baja -una diputada socialista declaraba: "Yo sigo la política de contestar a los de mi circunscripción o a los que necesitan algo de mis principales ocupaciones por las comisiones en que trabajo"-. Incluso, algunos diputados comentaban la utilidad de algunos de los mensajes enviados por ciudadanos; en esta línea un diputado de CiU indicaba: "A menudo hemos podido proponer iniciativas parlamentarias como resultado de las propuestas que personas en concreto nos han hecho llegar".

Lo que sí se aprecia en general en las respuestas de los diputados es su malestar por el volumen de correos recibidos como mensajes en cadena, remitidos por colectivos o ciudadanos que replican un mismo texto, con una misma demanda. Así lo manifiesta en su respuesta una diputada socialista: "El problema es que a menudo, en lugar de establecer una comunicación personal, lo que muchos internautas hacen es abusar y enviar un 'spam' continuo que impide precisamente esta comunicación".

Finalmente, solo 2 de los 97 diputados que respondieron pidieron explícitamente el voto para su formación política: PSOE y CiU.

\section{Reflexiones finales en torno al uso del e-mail para la comunicación directa entre ciudadanos y dipu- tados}

La denominada ciberdemocracia se concreta en la incorporación de las nuevas tecnologías de la información a la vida política y aspira a que los ciudadanos comunes puedan tener un papel más activo en las cuestiones públicas, por lo que necesitan una oportuna información y las herramientas necesarias para fomentar la participación. En la práctica, la red ofrece algunas posibilidades a los ciudadanos de a pie, 
principalmente a los que están interesados en la política, para participar en la vida pública. Pero todavía existen muchas limitaciones para que estas oportunidades puedan concretarse: bien por la responsabilidad del propio ciudadano o bien porque los gobernantes siguen mostrando más interés en la población como conjunto -fundamentalmente, a través de los medios de comunicación tradicionales y de las herramientas de Internet dirigidas a públicos amplios- que en la comunicación individual o personalizada con interacción -que permite las nuevas tecnología a través de algunas herramientas como, por ejemplo, el correo electrónico-.

Esta idea queda manifiesta en esta investigación: mientras las élites son el sector que más han aprovechado hasta ahora algunas de las posibilidades comunicativas mediante sus sitios web, páginas de candidatos en campaña electorales y otras iniciativas que han empezado a explotar las posibilidades abiertas a nuevas formas de publicidad y propaganda política, solo se aprecian tímidos avances en materia de diálogo real con grupos y ciudadanos por parte de los representantes (Dader, 2009; Dader, Vizcaíno, Campos y Cheng, 2010).

Ciertamente, el correo electrónico es, para la mayoría de los diputados, una herramienta conocida que gestionan bien personalmente bien a través de un asistente, pero a pesar de ello, el índice de respuesta por parte de los parlamentarios a un mensaje remitido por un supuesto ciudadano común queda todavía muy lejos del 50\% tanto del total de diputados de la Cámara como del grupo que publica una dirección electrónica de contacto (Dader y Campos, 2006). Casi una década después de que los primeros diputados empezaran a publicitar su $e$-mail y a responder a los mensajes ciudadanos, el hecho incontestable sigue siendo que el índice de respuesta que los ciudadanos pueden obtener de los parlamentarios del Congreso por correo electrónico es muy minoritario. Y ni siquiera parece afectar que sea en período de campaña electoral cuando el diputado reciba ese mensaje ciudadano.

Como cabría esperar, salvo escasas excepciones, la respuesta proviene generalmente de los parlamentarios de segunda línea. Tal y como habían comprobado Dader y Campos (2006), los políticos de primera línea disponen de menos tiempo o, sencillamente, no se toman la molestia de responder a los correos electrónicos remitidos por ciudadanos anónimos. Sobre este hecho, no obstante, hay que considerar que al haberse efectuado la consulta en la fase final de la campaña electoral es probable que los líderes principales y diputados de mayor relieve estuvieran demasiado inmersos en sus respectivas campañas y les resultara más difícil encontrar tiempo para responder su correo de la dirección parlamentaria. Aun así, conviene recordar que la medición realizada por Dader y Campos a finales de 2004, no coincidía con período electoral alguno y tampoco en ese caso las "celebridades parlamentarias" se prodigaron demasiado. En cualquier caso, resulta llamativo que el correo electrónico -a diferencia de las weblogs, páginas de candidato, entre otras herra- 
mientas de Internet (Dader, 2009) - sigue siendo de interés secundario para el conjunto de los representantes, a pesar de que estudios recientes revelan -al contrario de lo que ponen de manifiesto algunos de los diputados- que un escaso número de ciudadanos escriben a sus gobernantes (Anduiza et al., 2010).

Para tratar de entender el bajo índice de respuesta de los parlamentarios a los ciudadanos cabe tener presente algunos de los inconvenientes que el uso de esta herramienta tiene para los diputados y que apuntaron años antes Owen, Davis y Strickler (1999) en el caso norteamericano, pero con plena aplicación al caso español:

Los diputados no tienen capacidad para responder a los mensajes electrónicos recibidos: ni los grupos parlamentarios, ni sus partidos, ni tampoco las asambleas han previsto asistentes destinados a ayudar al parlamentario en la gestión de estas nuevas herramientas. Los representantes en las cámaras gestionan, en la medida de sus posibilidades -con o sin ayuda de sus asistentes-, estas nuevas herramientas al mismo tiempo que se ocupan de las tareas tradicionales.

Los parlamentarios reciben un flujo masivo de mensajes en cadena o spam cuyos autores no pretenden una respuesta personalizada sino mostrar una opinión contraria a cuestiones tratadas en la asamblea. Generalmente son mensajes que no proceden de electores o ciudadanos de sus circunscripciones y los parlamentarios consideran, en su mayoría, una pérdida de tiempo atender a estos correos.

A estas razones habría que añadir la idea planteada por Cardoso, Cunha y Nascimento (2003), que también resulta real en el caso español, cuando afirman que por parte de los parlamentarios se verifica un cierto desinterés y desconfianza de las potencialidades de esta herramienta argumentando, para ello, razones vinculadas a factores internos del propio parlamento, unido a la pervivencia del sistema de medios de comunicación tradicionales con predominio de la televisión, una cultura política débil o desinteresada por parte de los ciudadanos.

Al margen de este desinterés manifiesto por la mayoría de los diputados con el correo electrónico como vía de interacción con los ciudadanos, los resultados de esta investigación ponen de manifiesto la existencia de un grupo de parlamentarios en el Congreso que muestran una gran implicación con el e-mail -y seguramente con otras herramientas de Internet-para fomentar la comunicación directa con los ciudadanos, que leen y responden a los mensajes e incluso facilitan otros medios -como puede ser su número de teléfono móvil- para proseguir la comunicación. Por todo ello, es preciso considerar estas acciones de interacción vía $e$-mail como un punto de arranque que, aunque con un desarrollo temporal lento, se mantiene desde el año 1999 para una indispensable transformación futura de la institución parlamentaria en la que se ejerciten nuevas formas de implicación ciudadana en la actividad parlamentaria. 


\section{Referencias bibliográficas}

Anduiza et al. (2010): “Los usos políticos de Internet en España”, en Revista Española de Investigaciones Sociológicas (REIS), $\mathrm{n}^{\circ}$ 7. Madrid, Centro de Investigaciones Sociológicas, pp. 133-146.

Campos Domínguez, Eva (2009): El desarrollo de la ciberdemocracia en el Congreso de los Diputados. La comunicación e interacción entre ciudadanos y parlamentarios a través de Internet (2004-2008), tesis doctoral dirigida por DADER, José Luis, Madrid: Facultad de Ciencias de la Información, Universidad Complutense de Madrid.

Cardoso, Gustavo; Cunha, Carlos \& Nascimento, Susana (2003): “O parlamento portugués na construção de uma democracia digital”, en Sociologia, problemas e práticas, no 42. Lisboa: CIES-ISCTE / CELTA, pp. 113-140.

Carter, Matt (1999): "Speaking up in the Internet age: Use and value of constituent e-mail and Congressional Web-sites", en: Parliamentary Affairs, vol. 52, no 3, pp. 464-479.

Chappelet, Jean-Loup (2004): “The appropriation of e-mail and the Internet by members of the Swiss Parliament”, en: Information Polity. The International Journal of Government \& Democracy in the Information Age. Amsterdam: IOS Press, vol. 9, no.1/2, pp. 89-102.

Chen, Peter (2004): "A Comparative Analysis of Political Email Lists", en: Australian Electronic Governance Conference. Melbourne, Victoria: Centre for Public Policy, University of Melbourne, 14 y 15 de abril. Disponible en: http://www.publicpolicy.unimelb.edu.au/egovernance/ConferenceContent.html\#peterchen [Última consulta: 20 enero 2010].

Dader, José Luis (2001): “La ciberdemocracia posible: Reflexión prospectiva a partir de la experiencia en España”, en CIC. Cuadernos de información y comunicación, n ${ }^{\circ}$. Madrid: Universidad Complutense de Madrid, pp. 177-220.

Dader, José Luis (2003): “Ciberdemocracia y ciberparlamento: El uso del correo electrónico entre los parlamentarios españoles y los ciudadanos comunes (1999-2001)”, en Telos, Cuadernos de comunicación, tecnología y sociedad, nº 55, pp. 86-96.

Dader, José Luis \& CAMPOS, Eva (2006): "Internet parlamentario en España (1999-2005): los recursos para el contacto ciudadano y su uso, con una comparación europea”, en ZER, Revista de Estudios de Comunicación, n 20 . Bilbao: Universidad del País Vasco, pp. 105-132.

Dader , José Luis (2009): “Ciberpolítica en los websites de partidos políticos. La experiencia de las elecciones de 2008 en España ante las tendencias transnacionales”, en Revista de Sociologia e Politica, vol. 17, nº 34. Curitiba: Universidade Federal do Paraná, pp. 45-62.

Dader , José Luis; VIZCAÍNO, Ricardo; CAMPOS, Eva \& CHENG, Lifen (2010): “Las webs de los partidos españoles durante la campaña para las Elecciones Generales de 2008: pautas de cibermarketing con pocos signos de ciberdemocracia”, en SAMPEDRO, Víctor et al.: Ciberdemocracia en la campaña electoral 2008 en España, en imprenta.

Elms, Alan C. (1982): “Keeping deception honest: Justifying conditions for social scientific research strategems”, en BEAUCHAMOS, R.R. et al (eds.): Ethical issues in social science research. Baltimore, MD: Johns Hopkins University Press, pp. 232-245. 
Frau-Meigs, Divina (2001): “La democratie entre représentation et participation @ Internet.gov”, en SERFATY, Viviane (ed.): L'Internet en politique, es Etats-Unis à l'Europe. Strasbourg: Presses Universitarires de Strasbourg.

Frick, María Magdalena (2005): "Parliaments in the digital Age. Exploring Latin America", en e-DC Working Papers. e-Democracy Centre. Ginebra: University of Geneve.

López García, G. (2005): Modelos de comunicación en Internet. Valencia: Tirant Lo Blanch.

López, X. (2008): “La participación en los diarios digitales: similitudes y diferencias entre los cibermedios europeos y brasileños". En: VI Encontro nacional de Pesquisadores em Jornalismo. Universidade Metodista de Sao Paulo, noviembre de 2008. Disponible en: http://sbpjor.kamotini.kinghost.net/sbpjor/admjor/arquivos/coordenada4xoselopez.pdf [Última Consulta: 01/06/2010].

Norris, Pippa (2001): Digital Divide: Civic Engagement, Information Poverty and the Internet Worldwide. Cambridge University Press.

Owen, Diana; DAVIS, Richard \& STRICKLER, Vicent James (1999): “Congress and the Internet”, en: The Harvard International Journal of Press/Politics, vol. 4, nº2, pp. 10-29.

Sánchez González, M. (2010): “Efecto de la tecnología sobre la participación de las audiencias”. En: CABRERA GONZÁLEZ, M.A. (coord.): Evolución tecnológica y cibermedios. Zamora: Comunicación Social, Ediciones y Publicaciones.

Tucker, Kerrie (2004): "A Matter of Contempt - The Unauthorised Diversion and Receipt of a Member's e-mails", en Australian Electronic Governance Conference. Melbourne, Victoria: Centre for Public Policy, University of Melbourne, 14 y 15 de abril. Disponible en: http://www.public-policy.unimelb.edu.au/egovernance/papers/38_Tucker.pdf [Última consulta: 22 de enero de 2010].

Wimmer, Roger D. \& DOMINICK, Joseph R. (1996): La investigación científica de los medios de comunicación: una introducción a sus métodos (v.o. 1994). Barcelona: Bosch. 
